\section{Réformes des études et dimensions communautaires}

Parmi les orientations prises par la réforme des études à la Faculté de médecine de Genève, Chastonay et coll. nous présentent dans leur article [1] un des aspects les plus fascinants: l'enseignement des dimensions communautaires. A en lire cet article, il s'agit d'une véritable somme de savoir supplémentaire à laquelle les étudiants se voient confrontés: les sciences «populationnelles" comprenant entre autre l'épidémiologie, l'assurance de qualité des soins, l'économie de la santé; les sciences humaines touchant aux questions éthiques, historiques et légales de la médecine; enfin, des stages en communauté, p.ex. dans un cabinet d'un médecin de premier recours, sont organisés. Il est question de complexité bio-psycho-sociale, d'esprit critique vis-à-vis de la théorie et de la pratique de la médecine, de mieux saisir la complexité de la souffrance humaine.

En quelque sorte, nous assistons à un renversement de tendance. Toutes ces choses qui tournent autour de la médecine - l'Ethique, l'Histoire, les méthodologies de la Science - qu'on saisit mal et dont l'intérêt pour la pratique quotidienne n'est pas toujours évident sont bien souvent relégués aux préoccupations disons d'un âge professionnel plus mûr, la retraite seule laissant le loisir d'une réflexion approfondie. Il est vrai que beaucoup de médecins abordent leur vie professionnelle dans l'impatience d'agir, de faire quelque chose. Et ils ont raison, car ce sont les possibilités d'intervenir directement sur les patients et leurs maladies qui fascinent le plus, la médecine restant avant tout un métier pratique, dans la plupart des cas manuel. Or, c'est justement le plus actif, le plus interventionniste qui sera au cours de sa carrière confronté aux problèmes éthiques les plus difficiles (soins intensifs, transplantation, chirurgie oncologique, etc.). En défaut d'enseignement à propre- ment parler, ce sera l'expérience acquise au fil du temps qui formera une éthique individuelle qui n'est certes pas la plus mauvaise mais qui ne se transmettra que par l'exemple que l'un ou l'autre en tirera et qui surtout laissera seul devant le doute sur la justesse des actes dont le seul fondement aura été une réflexion personnelle. De cette évidence est né le désir d'enseigner l'éthique, c'est-à-dire de créer un savoir transmissible dont pourrait profiter le médecin du tout début de sa vie professionnelle. C'est donc chose faite et il faut le saluer.

Cependant, les nouvelles matières d'enseignement ne sont non seulement d'intérêt pour le médecin en tant qu'individu mais révèlent par ailleurs une évolution importante de la médecine en tant que science. Toute science, au cours de son évolution, tend à réfléchir sur ce qu'elle fait et ce qu'elle dit. Or pour ce faire, le langage technique ne suffit plus, il n'est pas apte à se décrire lui-même, de nouvelles notions deviennent nécessaires, une méta-science avec son langage propre doit se constituer [2]. De toute évidence, nous vivons une époque de la médecine qui essaye de mieux comprendre ses bases méthodologiques et d'en établir un système de règles. L'evidence-based medicine en est un exemple, peut-être encore quelque peu balbutiant. Dans ce contexte, l'intégration des sciences humaines en général dans la médecine pourrait ouvrir de nouvelles voies vers un raisonnement plus rigoureux sur ce qui fonde et ce qu'est la médecine. Ouvrir l'esprit des étudiants à ces perspectives est peut-être le défi le plus important des réformes en cours. Souhaitons leur un succès durable.

Dr Markus Trutmann Rédacteur en chef

1 Chastonay P, Vu NV, Stalder H et le Groupe Concept Didactique de la Dimension Communautaire. Enseignement des Dimensions Communautaires dans le cadre de la réforme des études de médecine de l'Université de Genève. Bull Méd Suisse 2000; 81:875-9.

2 Bochenski IM. Die zeitgenössischen Denkmethoden. 10. Auflage. Tübingen, Basel: Francke Verlag; 1993. 